\title{
In vivo growth of Epstein-Barr virus transformed B cells with mutations in latent membrane protein 2 (LMP2)
}

\author{
R. Rochford ${ }^{1}$, C. L. Miller ${ }^{2, *}$, M. J. Cannon ${ }^{3}$, K. M. Izumi ${ }^{2}$, E. Kieff ${ }^{2}$, and R. Longnecker ${ }^{4}$ \\ ${ }^{1}$ Department of Epidemiology, University of Michigan, Ann Arbor, Michigan, \\ U.S.A., ${ }^{2}$ Virology Program, Departments of Microbiology and Molecular \\ Genetics and Medicine, Harvard Medical School, Boston, Massachusetts, \\ U.S.A., ${ }^{3}$ Department of Microbiology and Immunology, University of \\ Arkansas for Medical Sciences, Little Rock, Arkansas, U.S.A. \\ ${ }^{4}$ Department of Microbiology - Immunology, Northwestern University \\ Medical School, Chicago, Illinois, U.S.A
}

Accepted October 15, 1996

\begin{abstract}
Summary. Epstein-Barr virus (EBV) causes infectious mononucleosis in adolescents and is associated with malignant B lymphocyte proliferation in AIDS patients, patients undergoing immune suppression for organ transplantation, and SCID mice. In vitro, EBV transformed, latently infected lymphoblastoid B cell lines (LCLs) contain EBV episomes and express nine virus encoded proteins. Six are nuclear proteins (EBNAs) and three are the integral membrane proteins, LMP1, LMP2A, and LMP2B. To determine if LMP2 was essential for in vivo growth, SCID mice were injected with LCLs containing wild-type EBV $\left(\mathrm{LMP}^{+}\right)$or with LCLs transformed with EBV containing mutations in either LMP2A or LMP2B $\left(\mathrm{LMP}^{-}\right)$. SCID mice injected with the $\mathrm{LMP}^{+}{ }^{+}$or LMP2 ${ }^{-}$ LCLs were monitored for tumor development, length of time to tumor development, and phenotypic characterization of the resulting tumors. No difference was observed in any of the above parameters between $\mathrm{LMP}^{+}$and $\mathrm{LMP}^{-}$LCLs demonstrating that LMP2 is not essential for the in vivo growth of EBV transformed B lymphocytes in SCID mice.
\end{abstract}

\section{Introduction}

Epstein-Barr virus (EBV) is a human herpesvirus which latently infects and transforms human primary B lymphocytes in vitro and, following primary infection in vivo, $\mathrm{EBV}$ is maintained as a latent infection in B cells (for review see $[17,30])$. EBV is associated with several human cancers including Burkitt's

* Present address: Millennium Pharmaceutical Inc., Cambridge, MA, U.S.A. 
lymphoma, nasopharyngeal carcinoma, and Hodgkin's disease in normal individuals (for review see [17,30]). In immunocompromised patients, EBV is associated with a variety of proliferative diseases including B cell lymphoproliferative disorders (LPDs), oral hairy leukoplakia (OHL), and smooth muscle tumors (for review see [17, 30]).

In vitro, EBV transformed latently infected B lymphocytes (LCLs) express nine viral encoded proteins from the episomally maintained viral genome: the Epstein-Barr nuclear antigens (EBNAs) EBNA1, EBNA2, EBNALP, EBNA3A, EBNA3B, and EBNA3C and the integral latent membrane proteins (LMPs) LMP1, LMP2A, and LMP2B (for review see [17, 30]). EBNA1, LMP1, LMP2A, and LMP2B are the only latently expressed proteins consistently detected in EBV proliferative disorders in vivo [3, 5, 7, 11, 12, 15, 35, 37, 46, 47, 51, 52]. Similarly, EBNA1 and LMP2A messages are the only EBV-specific messages detected in PCR analysis of B lymphocytes from individuals harboring latent EBV infections [39, 49], thus implicating an important role for these gene products in in vivo EBV infection and related disease.

EBV recombinant molecular genetic experiments have demonstrated that neither LMP2A or LMP2B is a mediator of the B lymphocyte growth transformation that accompanies latent in vitro infection [23-25]. However, analysis of LCLs infected with LMP2 mutants has specified a role for LMP2A in modulating EBV latency in EBV infected LCLs [29]. The LMP2A primary amino acid sequence includes 119 amino acids at the amino terminus, twelve hydrophobic domains of a least 16 amino acids, each of which traverses the plasma membrane, and a 27 amino acid carboxyl terminal domain (Fig. 1, [18, 19, 45]). The 119 amino acid cytoplasmic amino terminal domain, unique to LMP2A, is essential for the function of LMP2A. EBV ${ }^{+} \mathrm{LMP}_{2} \mathrm{~A}^{+} \mathrm{LCLs}$ are blocked in surface immunoglobulin (slg) stimulated calcium mobilization, and lytic activation as compared to $\mathrm{EBV}^{+} \mathrm{LMP}^{-\mathrm{A}^{-}}$LCLs [29]. Given that LMP2A is detected in latently infected $\mathrm{B}$ cells in vivo, a possible role for LMP2A in the growth of latently infected B cells in vivo can be argued.

B cell lymphomas develop in SCID mice following reconstitution with peripheral blood lymphocytes (PBLs) from EBV negative donors with subsequent infection with EBV [6, 36], or by reconstitution with PBLs from EBV seropositive individuals and by transfer and subsequent growth of LCLs in SCID mice [6, 32-34, 36, 40, 44]. These tumors are of human B cell origin, contain EBV genomes and express EBV latent genes, and express a similar pattern of cell surface markers as that seen in LPDs in immunocompromised individuals $[40,44]$. The phenotype of these B cell tumors in SCID mice differs from that seen when the same EBV transformed cells are grown in tissue culture $[13,40-42]$. In particular, the B cell lineage activation antigens CD20 and CD23 are reduced in SCID tumors [13,40], and there is an increase in CD38 expression [40] relative to the same LCLs grown in vitro. CD23 is expressed on mitogen stimulated or EBV-infected B lymphocytes, but not on mature plasma cells $[48,50]$. CD38 is found only on pre-B cells and plasma cells, but not on activated $\mathrm{B}$ cells $[1,50]$. Also associated with the SCID tumors is a large production of 
human $\operatorname{Ig}[13,38]$ and a reduction in the expression of EBNA2 and LMP1 $[40,42]$. Thus, there is a shift to a more differentiated plasma cell when EBV infected lymphocytes are grown in, or isolated from, SCID mice relative to the same infected B lymphocytes grown in vitro. In addition, previous studies have shown EBNA2 is a critical determinant for tumor growth in SCID mice [9]. Thus, SCID mice represent a potentially useful model system for exploring the in vivo growth characteristics of in vitro transformed B lymphocytes latently infected with EBV containing mutations in LMP2.

In this report, three sets of specifically mutated EBV recombinants have been used to evaluate the role of LMP2A and LMP2B in B cell growth following transfer of LCLs into SCID mice. The mutations stop translation of LMP2A after the 19th codon (19S) [23], after the 260th codon of LMP2A or the 141st codon of LMP2B (260S) [24], or delete codons 120 to 260 of LMP2A or codons 1 to 141 of LMP2B (ES) [25] (Fig. 1, 19S, 260S, and ES). EBV with each of these mutations is able to transform primary $\mathrm{B}$ lymphocytes in vitro and the transformed B lymphocytes are indistinguishable in their growth in vitro from cells transformed by wild-type EBV as measured by growth at low cell densities in media supplemented with lower amounts of serum or when cells are grown in soft agar [23-25]. LCLs infected with each of the LMP2 mutations were not altered in their in vivo growth properties in SCID mice when compared to LCLs infected with wild-type EBV.

\section{Results}

To verify that LMP2A and LMP2B are expressed in B cell tumors which develop in SCID mice following injection of EBV transformed LCLs, $3 \times 10^{6}$ wild-type infected LCLs which had been maintained in vitro for 6 months were injected into two SCID mice. Six weeks after injection, the mice were sacrificed and RNA was prepared as described [8] and subjected to a multiprobe ribonuclease protection assay as previously described $[16,40,42]$. The EBNA2, LMP1, EBNA1 and cellular ribosomal protein (rp) L32 riboprobes used for the protection assay have previously been described [40, 42]. The LMP2 riboprobe, nucleotides 545-706 [45], was designed to detect both the LMP2A and LMP2B mRNAs and was made as previously described [42]. Transcripts for each of the EBV latent genes were readily detected in tumors prepared from each of the injected SCID mice (Fig. 2).

To investigate if LMP2 may be a determinant of in vivo growth in SCID mice, lytic replication was induced in LCLs infected with the 19S, 260S, and wild-type EBV (Fig. 2, WT, 19S, 260S) by treatment with TPA and electroporation with pSVNaeZ as previously described [23]. The plasmid pSVNaeZ encodes the immediate-early transactivor Z ta of lytic replication and induces lytic phase in latently infected LCLs [10, 14, 23, 43]. B lymphocytes from two separate donors were purified as previously described and infected with virus from the induced LCLs [26]. Different donors were used for the establishment of LCLs infected with the $19 \mathrm{~S}$ or $260 \mathrm{~S}$ LMP2 mutant viruses. Wild-type EBV 


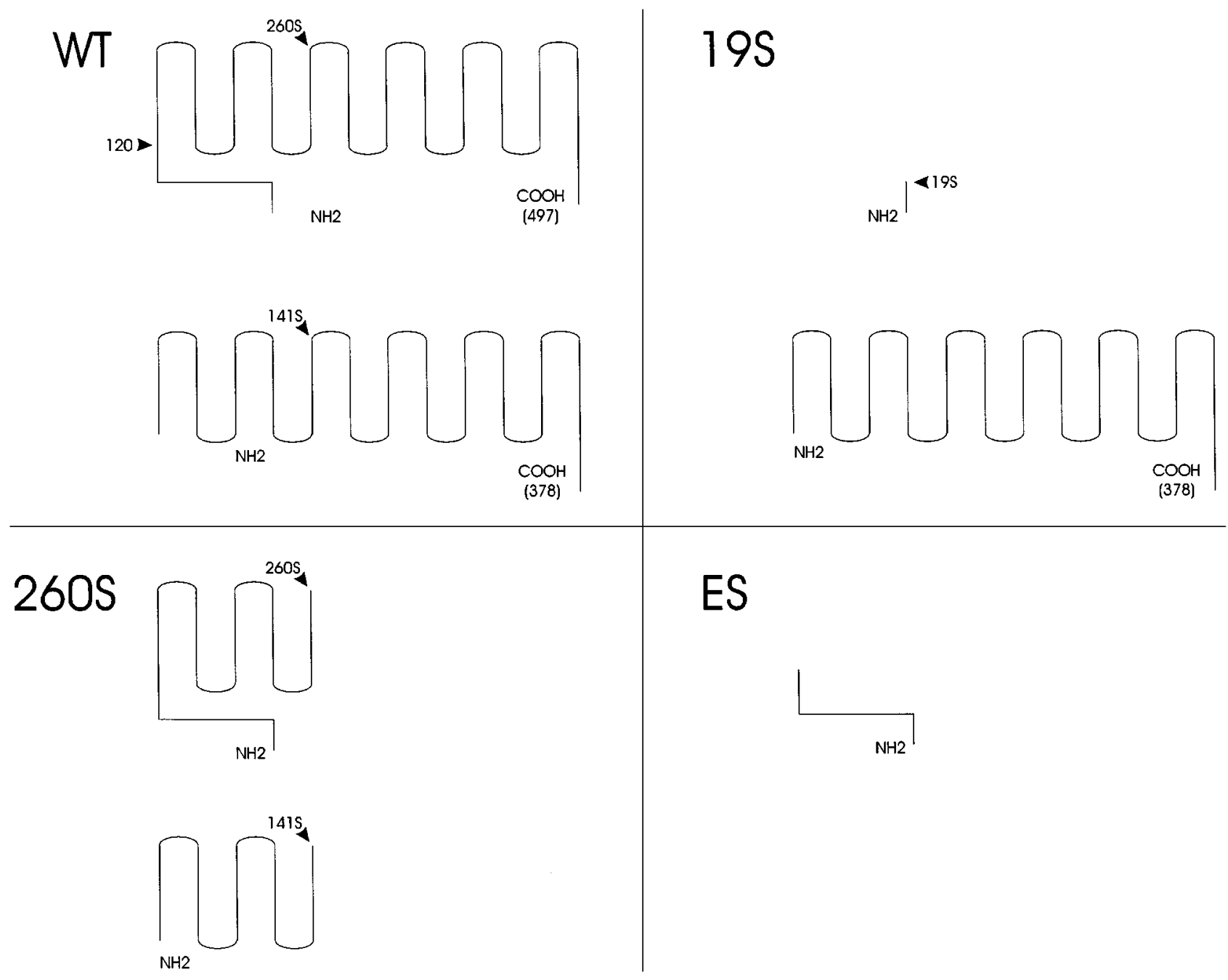

Fig. 1. Schematic representation of the predicted structure of LMP2A (top of each panel) and LMP2B (bottom of each panel) and the various mutants in the cell plasma membrane in EBV infected B cells. $W T$ The wild-type LMP2A and LMP2B proteins are indicated. Sites of insertion of the translation stop codons are indicated as well as the relevant amino acid numbers. 19S Expected LMP2 protein products in 19S mutant infected LCLs [23]. 260S Expected LMP2 truncation products in 260S mutant infected LCLs. Expression of the 260S truncation product was previously confirmed and was altered in its subcellular location when compared to wild-type infected LCLs as described in the text [24]. ES Expected LMP2 protein products in ES mutant infected LCLs. The deletion of the EBV genomic sequences does not leave the LMP2A coding domain in frame. The LMP2A amino terminal truncation product which may be expressed was not detected in ES infected LCLs [25]

infected LCLs from the same donors were derived in parallel. LCLs emerged 3 to 6 weeks after infection and were expanded. Ten different LCLs for each mutant and appropriate donor matched wild-type infected LCLs were identified and expanded. The presence or absence of the LMP2 mutation was confirmed by 


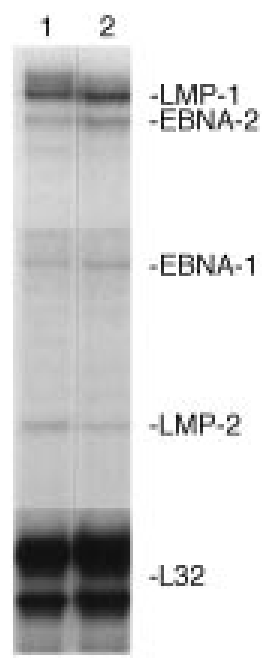

Fig. 2. EBV latent gene expression in SCID tumor cells. A multiprobe ribonuclease protection assay was performed to detect viral LMP1, LMP2, EBNA1, and EBNA2 and cellular rpL32 in SCID tumor cells. RNA from $1 \times 10^{6}$ cells was analyzed in each lane. 1 and 2 represent RNA extracted from two different wild-type infected LCL derived tumors

PCR $[23,24]$. After establishment of mutant and wild-type LCLs in vitro (within 60 days post initial outgrowth), the LCLs were analyzed for their ability to induce tumors in SCID mice. LCLs were washed once with sterile phosphate buffered saline (PBS), resuspended in PBS, and $5 \times 10^{6}$ cells per LCL were injected intraperitoneally into four-week-old male SCID mice. Recipient SCID mice were maintained in micro-isolator cages in a sterile environment. Mice were monitored daily for signs of LCL outgrowth and related illness (signs include weight loss, inactivity, ruffled fur, abdominal swelling, and palpable abdominal masses). When clinical symptoms became apparent, or when the mice were in obvious distress, the animals were sacrificed and analyzed for signs of lymphoproliferative disease, organ infiltration, and tumor formation.

Nine of ten independent 19S LMP2 mutant infected LCLs were capable of initiating tumors in SCID mice at a rate that was indistinguishable from the nine of ten wild-type control LCLs that also induced tumors (Table 1). Most animals were sacrificed when illness and/or palpable tumors became apparent. In all cases there were signs of lymphoproliferative disease and tumorigenesis, which included visible tumor masses, organ infiltration, abdominal ascites, or subcutaneous tumors at the site of injection. Nine of ten independent 260S LMP2 mutant EBV infected LCLs were also found to efficiently induce lymphoproliferative disease and tumor formation following injection into SCID mice with similar kinetics as 19S or the 19S wild-type control infected LCLs (compare 42.4 days with 45.4 days and 39.2 days, Table 1). The wild-type control LCLs (Table $1, \mathrm{WT}^{\mathrm{d}}$ ) derived in parallel with the $260 \mathrm{~S}$ LCLs had a somewhat shorter mean number of days to tumor formation than the other LCLs (compare 27.9 days to 42.4 days, 45.4 days, and 39.2 days, Table 1). The SCID mice that showed no clinical signs of LCL-related disease were sacrificed after 52 days and found to have no internal signs of lymphoproliferative disease or tumorigenesis. The 
Table 1. Tumor development in SCID mice injected with 19S, 260S, and control wild-type EBV infected LCLs

\begin{tabular}{ll}
\hline Virus $^{\mathrm{a}}$ & Mean number of days to tumor formation \\
\hline $19 \mathrm{~S}$ & $39.2\left(52,30^{*}, 28,36,33,40,46^{*}, 48,40\right)$ \\
$\mathrm{WT}^{\mathrm{c}}$ & $45.4\left(52,52,36,52,48^{*}, 35,52,35,47^{*}\right)$ \\
$260 \mathrm{~S}$ & $42.4\left(40,40,52,33^{*}, 52,47,47,40,31^{*}\right)$ \\
WT $^{\mathrm{d}}$ & $27.9\left(26,23^{*}, 33,27,26,27,24^{*}, 34,31\right)$
\end{tabular}

* Mice which died before being sacrificed

${ }^{a}$ Wild-type and mutant infected LCLs were constructed with the parental P3HR1 virus and are more fully described in [23, 24]

${ }^{\mathrm{b}} 10$ four week old male SCID mice were each injected with $5 \times 10^{6}$ cells of 1 of 10 recently derived, independent EBV infected LCLs. Numbers in parentheses are results for each mouse in the group which developed tumors, reflecting days until overt signs of illness or, where indicated $\left(^{*}\right)$, number of days until the animal died. One mouse from each group did not develop a tumor

${ }^{\mathrm{c}}$ Wild-type EBV infected LCLs derived in parallel from the same donor as the 19S LCLs

${ }^{\mathrm{d}}$ Wild-type EBV infected LCLs derived in parallel from the same donor as the 260S LCLs

absence of tumor development in $10 \%$ of the injected SCID mice is consistent with the occurrence of breakthroughs which occur in approximately $10 \%$ of SCID mice; these so-called leaky mice cannot be successfully reconstituted with human cells [2]. To confirm that the resulting tumors arising from the mice injected with LMP2 mutants contained the LMP2 mutation, PCR analysis was performed on LCLs established in vitro from tumor samples. No reversion was detected thus suggesting that the LMP2 mutations were maintained following in vivo growth (data not shown).

To further investigate the growth properties of LCLs infected with LMP2 mutant EBV the following experiment was performed. Two ES LCLs (Fig. 1, ES) and two wild-type LCLs which had been derived from the same donor cells and maintained in vitro for 3 to 6 months were evaluated for tumor formation in SCID mice following intraperitoneal injection. Four groups of four SCID mice each were injected with $3 \times 10^{6}$ of each wild-type or mutant infected LCL. Animals were monitored and sacrificed when illness and/or palpable tumors became apparent. All four animals injected with the ES5 LCL developed tumors within 33 days after injection with a mean of 29.5 days (Table 2). Three of four animals injected with the ES4 LCL developed tumors with a mean of 76 days (Table 2). Both wild-type LCLs caused tumors following injection. WT3 LCL, similarly to the ES5 LCL, developed tumors within 33 days with a mean of 26 days (Table 2). In contrast, WT20 injected animals developed tumors within 76 days with a mean of 60.75 days (Table 2) similar to the rate seen with ES4 LCL. 
Table 2. Tumor development in SCID mice injected with ES and control wild-type EBV infected LCLs

\begin{tabular}{ll}
\hline Virus $^{\mathrm{a}}$ & Mean number of days to tumor formation \\
\hline ES4 $^{\mathrm{c}}$ & $76\left(68^{*}, 92,68\right)$ \\
ES5 & $29.5\left(28,30^{*}, 33,27\right)$ \\
WT3 & $26\left(23^{*}, 24,24,33\right)$ \\
WT20 & $60.75\left(75^{*}, 48^{*}, 76,44\right)$
\end{tabular}

* Mice which died before being sacrificed

${ }^{a}$ Wild-type and mutant infected LCLs were constructed with the parental B95-8 virus and are more fully described in [25]. All LCLs were derived in parallel from the infection of the same donor cells

${ }^{b} 4$ four week old male SCID mice were each injected with $3 \times 10^{6}$ cells of each LCL. Numbers in parentheses are results for each mouse in the group which developed tumors, reflecting days until overt signs of illness, or where indicated $(*)$, number of days until the animal died

${ }^{\mathrm{c}}$ One mouse did not develop tumor

These results indicated that LCLs infected with EBV containing the ES mutation in LMP2 caused tumor formation in SCID mice following injection in a similar manner as observed with wild-type LCLs.

Previous observations had indicated that LCLs grown in vitro when injected into SCID mice down modulate the expression of the $\mathrm{B}$ cell activation markers CD20 and CD23 and upregulate the expression of the plasmacytoid marker CD38 [13, 40, 41]. Differences in the expression of CD23 and CD38 in LCLs or in tumor cells were determined for ES4, ES5, WT3, and WT20. Tumor cells were dispersed by collagenase treatment of minced tissue as previously described $[6$, 40]. Flow cytofluorometric (FCF) analysis on a FACScan following immunostaining with FITC conjugated anti-CD23 mAb (BU38, The Binding Site, Birmingham, U.K.), and PE-conjugated anti-CD38 mAB (Becton Dickinson, Bedford, MA) was performed on tumor cells and LCLs grown in vitro as previously described $[6,40]$. The presence of contaminating mouse cells was excluded by staining tumor cells for HLA class I antigen with biotinylated anti-H-2K ${ }^{d}$ (Pharmingen, San Diego) followed by streptavidin-PerCP (Becton-Dickinson, Bedford, MA). Representative plots from the FCF analysis are shown in Fig. 2 and Table 3 is a summary of the mean fluorescent intensities (MFI) for the cells analyzed. As was previously described, CD23 expression decreased in wild-type tumor cells when compared to the same LCL grown in vitro. There was a reduction of the MFI from 714 to 199 for WT3 and 320 to 132 for WT20 (Table 3). There was no qualitative difference when the LMP2 mutant cells were analyzed. Reduction of the MFI of 668 to 110 for ES4 and 998 to 227 for ES5 was similar to that observed for the wild-type cells (Table 3). Also as previously described, CD38 expression increased in wild-type tumor cells when compared to the same LCL grown in vitro. Increase of MFI was from 131 to 631 for WT3 
Table 3. Mean fluorescence intensities for LCLs and tumor samples

\begin{tabular}{llc}
\hline & Marker $^{\mathrm{a}}$ & \\
\cline { 2 - 3 } Cell line & CD23 & CD38 \\
\hline WT3 & 714 & 134 \\
WT3 tumor & 199 & 631 \\
WT20 & 320 & 87 \\
WT20 tumor & 132 & 146 \\
ES4 & 668 & 267 \\
ES4 tumor & 110 & 587 \\
ES5 & 998 & 494 \\
ES5 tumor & 227 & 760 \\
\hline
\end{tabular}

${ }^{a}$ Expression of the $\mathrm{B}$ cell surface markers CD23 and CD38 were examined by $\mathrm{FCF}$ analysis as previously described $[6,40]$. In tumor samples, the presence of contaminating mouse cells were excluded by staining for HLA class I antigen

and 87 to 146 for WT20 (Table 3). The LMP2 mutant infected LCLs behaved similarly. For ES4 there was an increase of the MFI for CD38 from 267 to 587 and for ES5 an increase from 494 to 760 was observed (Table 3). These data indicate there was no differences in the differentiation in LMP2 mutant LCLs injected into SCID mice when compared to wild-type LCLs.

\section{Discussion}

Previous studies demonstrated that LCLs infected with LMP2 mutants grew equally well in vitro as wild-type infected LCLs as measured by growth at low cell densities in media supplemented with lower amounts of serum or when cells are grown in soft agar [23-25]. Analysis of LCLs infected with the LMP2 mutants has specified a role of LMP2A in maintaining EBV latency in vitro. The 119 amino acid cytoplasmic domain, unique to LMP2A, is essential for the function of LMP2A. EBV + LMP2A + LCLs are blocked in slg mediated calcium mobilization, tyrosine phosphorylation, and activation of lytic viral replication as compared to EBV + LMP2A- LCLs. LMP2A functions as a negative regulator of the Src family PTK lyn, and the Syk PTK, while LMP2B may regulate LMP2A function by modulating LMP2A function since it lacks the 119 amino acid cytoplasmic domain present in LMP2A. Truncation of LMP2A after the fifth transmembrane domain results in diffuse plasma membrane localization and an intermediate calcium phenotype when compared to EBV + LMP2A + and EBV + LMP2A- LCLs confirming the importance of the multiple membrane domains in the fully aggregated phenotype [21, 25]. Our model of LMP2A regulation of EBV latent infection hypothesizes that the LMP2A amino terminal domains, when aggregated and complexed with the Syk and the Src family PTKs, resembles crosslinked cell surface receptors in the plasma membrane of 

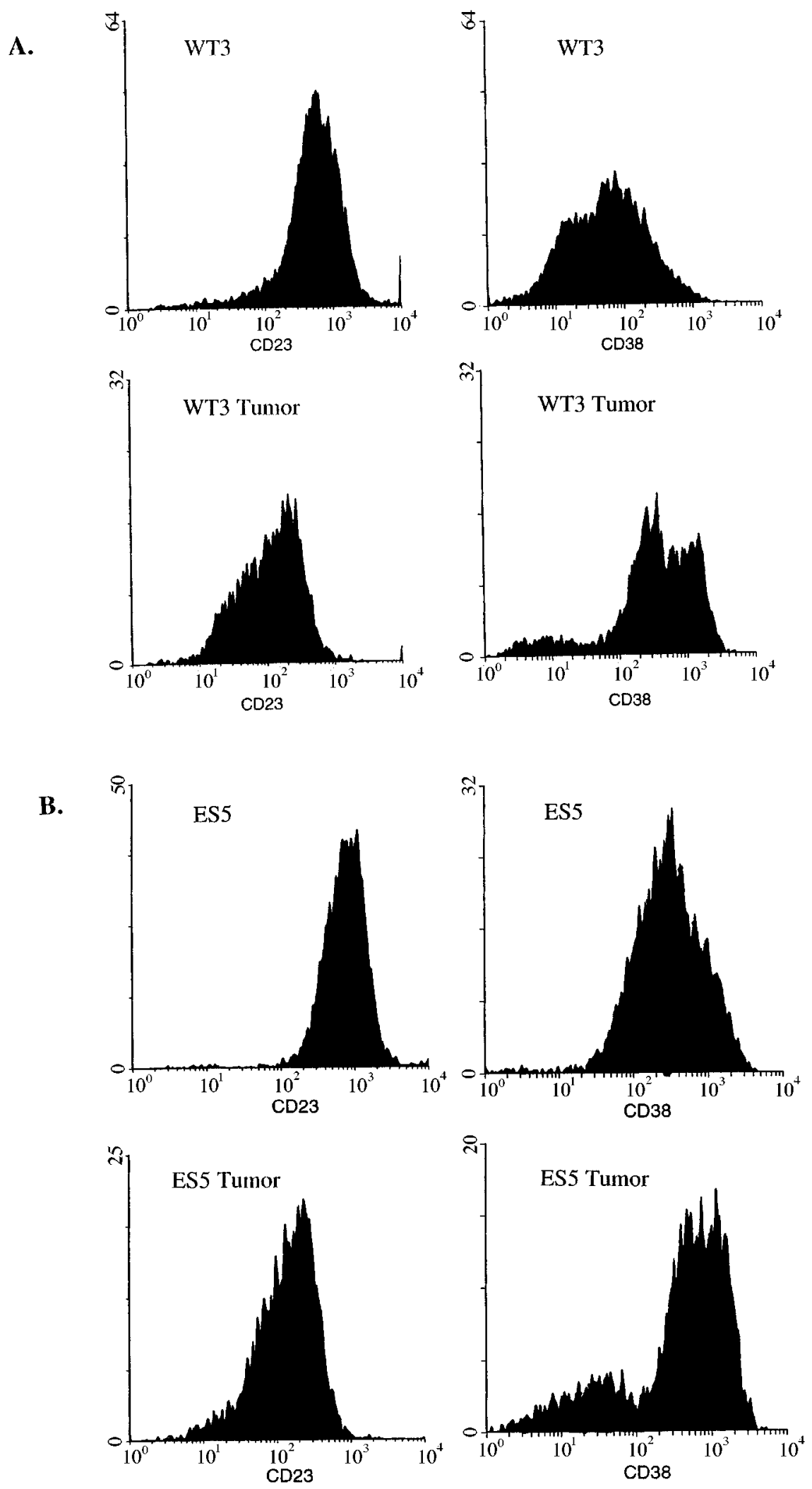

Fig. 3. Expression of the B cell markers CD23 and CD 38 in LCLs and tumor cells for wildtype (A) and ES infected (B) LCLs. Cells were prepared and subjected to FCF as described in the text 
EBV infected cells $[22,28]$. This results in down modulation of the enzymatic activities of the the Syk and Src family PTKs [27]. Other LMP2A associated proteins not yet identified may be equally affected. In immunoprecipitation studies, LMP2A associates with at least eight unidentified phosphoproteins of varying molecular weights $[4,20,21]$.

These observations and the identification of the LMP2A and EBNA1 mRNAs as the only EBV-specific mRNAs detected in normal individuals harboring EBV latent infections [39, 49], argue for an important role for LMP2 in vivo. In addition, it was demonstrated in this study that the LMP2 genes are expressed in SCID tumors following injection of wild-type infected LCLs. To begin to test for a role of LMP2 in vivo, three different LMP2 mutants were analyzed for in vivo growth in the SCID mouse model system of EBV LPD. The first mutant, 19S, expresses LMP2B but does not express LMP2A as the result of a termination codon inserted after LMP2A amino acid 19 [23]. Both LMP2A and LMP2B are truncated in the 260S mutation, LMP2A after 260 amino acids and LMP2B after 141 amino acids [24]. In the ES mutation, both LMP2A and LMP2B are deleted resulting in the absence of both proteins or detectable truncation products [25]. Using LCLs with each of the LMP2 mutations, it was determined that neither LMP2A or LMP2B is a key determinant for tumor growth in SCID mice as measured by days to tumor formation. In addition, it was shown that the LMP2 mutant LCLs behaved in a similar fashion to wild-type infected LCLs in regard to changes in expression of developmentally regulated $\mathrm{B}$ cell surface markers $\mathrm{CD} 23$ and CD38.

The absence of an effect of the LMP2 mutations in the SCID mouse model of EBV LPD is somewhat surprising. If LMP2 is important for maintaining latency in vivo the ability of LCLs transformed by the LMP2 mutants may have been expected to be severely hampered in their ability to form tumors in SCID mice. This would be a result of a larger fraction of cells in the LMP2 mutant infected cells undergoing lytic replication and thus susceptible to cell death induced by lytic virus replication. In normal hosts, only the transcripts of the LMP2A and EBNA1 mRNAs have been detected [39, 49] and the cells are in a nonactivated state [31]. In the SCID mouse model of EBV induced LPD, previous studies have shown that the tumors which arise consist of two distinct populations [13, 40,41]. One population in which only latent EBV transcripts are found and which express levels of CD23 and CD38 (CD23 $\left.{ }^{\text {int }} \mathrm{CD} 38^{\text {int }}\right)$ similar to that when the same LCLs are grown in vitro [42]. In these same $\mathrm{CD} 23^{\text {int }} \mathrm{CD} 38^{\text {int }}$ cells, there is a reduction in the expression of the EBNA1, EBNA2 and LMP1 transcripts [42]. In the other population, there is a reduction in the expression of CD23 (CCD23 ${ }^{\text {lo }}$ ) and higher levels of CD38 (CD38 $\left.8^{\text {hi }}\right)$ and only lytic transcripts are detected [42]. Further, in this $\mathrm{CD} 23^{\mathrm{lo}} \mathrm{CD} 38^{\mathrm{hi}}$ population, $87 \%$ of the cells were positive for gp350 mRNA expression by in situ hybridization [42]. Since neither of these populations represents the pattern of viral gene expression seen in normal latently infected human hosts, it may not be surprising that no difference was detected between wild-type and the LMP2 mutant infected LCLs in the SCID mouse model of EBV LPD. 
It is worth noting that SCID mice do not accurately mimic "normal" EBV human hosts in several ways. First, there are no functional $T$ cells present that are capable of applying immune regulatory pressure on the latently infected LCLs, which is likely to affect the pattern of latent gene expression and/or reactivation of lytic infection. Second, SCID mice are out of necessity maintained in a pathogen-free environment, and are therefore unlikely to encounter typical B-cell mitogens which might otherwise impact EBV gene expression or reactivation. We have hypothesized from our in vitro studies that the major role of LMP2A in latent EBV infection may be to prevent activation of lytic EBV replication by cell surface mediated signal transduction $[22,28]$. The likely importance of this function is in preventing lytic replication in latently infected B lymphocytes as they circulate in the peripheral blood, bone marrow, or lymphatic tissues where they might encounter antigens, super-antigens, or other ligands which could engage $\mathrm{B}$ cell receptors and could activate EBV lytic replication. Until better models of EBV latent infection in which only EBNA1 and LMP2A are expressed, it may be difficult to delineate the role of LMP2 in vivo using currently available in vitro models of in vivo EBV latent infection.

\section{Acknowledgements}

This research was supported by Public Health Service grants CA62234 (R.L.), CA47006 (E.K.), and CA63931 (M. J. C.). R.R. is a Special Fellow and R.L. is a Scholar of the Leukemia Society of America. R.L. is also a recipient of a grant from the Tobacco Research Council, USA.

\section{References}

1. Anderson KC, Park EK, Bates MP, Leonard RC, Hardy R, Schlossman SF, Nadler LM (1983) Antigens on human plasma cells identified by monoclonal antibodies. J Immunol 130: 1 132-1 138

2. Bosma GC, Fried M, Custer RP, Carroll A, Gibson DM, Bosma MJ (1988) Evidence of functional lymphocytes in some (leaky) scid mice. J Exp Med 167: 1016-1 033

3. Brooks L, Yao QY, Rickinson AB, Young LS (1992) Epstein-Barr virus latent gene transcription in nasopharyngeal carcinoma cells: coexpression of EBNA1, LMP1, and LMP2 transcripts. J Virol 66: 2 689-2 697

4. Burkhardt AL, Bolen JB, Kieff E, Longnecker R (1992) An Epstein-Barr virus transformation-associated membrane protein interacts with src family tyrosine kinases. J Virol 66: 5 161-5 167

5. Busson P, McCoy R, Sadler R, Gilligan K, Tursz T, Raab-Traub N (1992) Consistent transcription of the Epstein-Barr virus LMP2 gene in nasopharyngeal carcinoma. J Virol 66: 3 257-3 262

6. Cannon MJ, Pisa P, Fox RI, Cooper NR (1990) Epstein-Barr virus induces aggressive lymphoproliferative disorders of human B cell origin in SCID/hu chimeric mice. J Clin Invest 85: 1333-1 337

7. Chen CL, Sadler RH, Walling DM, Su IJ, Hsieh HC, Raab-Traub N (1993) Epstein-Barr virus $(\mathrm{EBV})$ gene expression in EBV-positive peripheral T-cell lymphomas. J Virol 67: 6303-6 308 
8. Chomczynski P, Sacchi N (1997) Single-step method of RNA isolation by acid guanidium thyocyanate-phenol-chloroform extraction. Anal Biochem 162: 156159

9. Cohen JI, Picchio GR, Mosier DE (1992) Epstein-Barr virus nuclear protein 2 is a critical determinant for tumor growth in SCID mice and for tranformation in vitro. J Virol 66: $7555-7559$

10. Countryman J, Jenson H, Seibl R, Wolf H, Miller G (1987) Polymorphic proteins encoded within BZLF1 of defective and standard Epstein-Barr viruses disrupt latency. J Virol 61: 3 672-3 679

11. Deacon EM, Pallesen G, Niedobitek G, Crocker J, Brooks L, Rickinson AB, Young LS (1993) Epstein-Barr virus and Hodgkin's disease: transcriptional analysis of virus latency in the malignant cells. J Exp Med 177: 339-349

12. Delsol G, Brousset P, Chittal S, Rigal HF (1992) Correlation of the expression of Epstein-Barr virus latent membrane protein and in situ hybridization with biotinylated BamHI-W probes in Hodgkin's disease. Am J Pathol 140: 247-253

13. Garnier JL, Cooper NR, Cannon MJ (1993) Low expression of CD20 and CD23 in Epstein-Barr virus-induced B cell tumors in SCID/hu mice. Am J Pathol 142: $353-358$

14. Grogan E, Jenson H, Countryman J, Heston L, Gradoville L, Miller G (1987) Transfection of a rearranged viral DNA fragment, WZhet, stably converts latent Epstein-Barr viral infection to productive infection in lymphoid cells. Proc Natl Acad Sci USA 84: $1332-1336$

15. Herbst H, Dallenbach F, Hummel M, Niedobitek G, Pileri S, Muller LN, Stein H (1991) Epstein-Barr virus latent membrane protein expression in Hodgkin and Reed-Sternberg cells. Proc Natl Acad Sci USA 88: 4 766-4 770

16. Hobbs MV, Weigle WO, Noonan DJ, Torbett BE, McEvilly RJ, Koch RJ, Cardenas GJ, Ernst DN (1993) Patterns of cytokine gene expression by CD4 + T cells from young and old mice. J Immunol 150: 3 602-3 614

17. Kieff E (1996) Epstein-Barr virus and its replication. In: Fields BN, Knipe DM (eds) Fundamental virology Raven Press, New York, pp 1 109-1 161

18. Laux G, Economou A, Farrell PJ (1989) The terminal protein gene 2 of Epstein-Barr virus is transcribed from a bidirectional latent promoter region. J Gen Virol 70: $3079-3084$

19. Laux G,Perricaudet M, Farrell PJ (1988) A spliced Epstein-Barr virus gene expressed in immortalized lymphocytes is created by circularization of the linear viral genome. Embo J 7: 769-774

20. Longnecker R (1996) Unpublished results

21. Longnecker R, Druker B, Roberts TM, Kieff E (1991) An Epstein-Barr virus protein associated with cell growth transformation interacts with a tyrosine kinase. J Virol 65: 3 681-3 692

22. Longnecker R, Miller CL (1996) Regulation of Epstein-Barr virus latency by latent membrane 2. Trends Microbiol 4: 38-42

23. Longnecker R, Miller CL, Miao X-Q, Marchini A, Kieff E (1992) The only domain which distinguishes Epstein-Barr virus latent membrane 2A (LMP2A) from LMP2B is dispensable for lyphocyte infection and growth transformation in vitro, and LMP2A is therefore nonessential. J Virol 66: 6 461-6469

24. Longnecker R, Miller CL, Miao X-Q, Tomkinson B, Kieff E (1993) The last seven transmembrane and carboxy terminal tail of Epstein-Barr virus latent membrane 2 (LMP2) are dispensable for lymphocyte infection and growth transformation. J Virol 67: 2 006-2 013 
25. Longnecker R, Miller CL, Tomkinson B, Miao XQ, Kieff E (1993) Deletion of DNA encoding the first five transmembrane domains of Epstein-Barr virus latent membrane proteins 2A and 2B. J Virol 67: 5068-5074

26. Marchini A, Tomkinson B, Cohen JI, Kieff E(1991) BHRF1, the Epstein-Barr virus gene with homology to $\mathrm{Bc} 12$, is dispensable for $\mathrm{B}-1 y m p h o c y t e$ transformation and virus replication. J Virol 65: 5 991-6000

27. Miller CL, Burkhardt AL, Lee JH, Stealey B, Longnecker R, Bolen JB, Kieff E (1995) Integral membrane protein 2 of Epstein-Barr virus regulates reactivation from latency through dominant negative effects on protein-tyrosine kinases. Immunity 2 : $155-166$

28. Miller CL, Lee JH, Kieff E, Burkhardt AL, Bolen JB, Longnecker R (1994) Epstein-Barr virus protein LMP2A regulates reactivation from latency by negatively regulating tyrosine kinases involved in slg-mediated signal transduction. Infect Agents Dis 3:128136

29. Miller CL, Lee JH, Kieff E, Longnecker R (1994) An integral membrane protein (LMP2) blocks reactivation of Epstein-Barr virus from latency following surface immunoglobulin crosslinking. Proc Natl Acad Sci USA 91: 772-776

30. Miller G (1990) Epstein-Barr virus. In: Fields BN, Knipe DM (eds) Virology. Raven Press, New York, pp 563-589

31. Miyashita EM, Yang B, Lam KM, Crawford DH, Thorley-Lawson DA (1995) A novel form of Epstein-Barr virus latency in normal B cells in vivo. Cell 80: 593-601

32. Mosier DE, Baird SM, Kirven MB, Gulizia RJ, Wilson DB, Kubayashi R, Picchio G, Garnier JL, Sullivan JL, Kipps TJ (1990) EBV-associated B-cell lymphomas following transfer of human peripheral blood lymphocytes to mice with severe combined immune deficiency. Curr Top Microbiol Immunol 166: 317-323

33. Mosier DE, Gulizia RJ, Baird SM, Wilson DB (1988) Transfer of a functional human immune system to mice with severe combined immunodeficiency. Nature 335 : $256-259$

34. Mosier DE, Picchio GR, Baird SM, Kobayashi R, Kipps TJ (1992) Epstein-Barr virus-induced human B-cell lymphomas in SCID mice reconstituted with human peripheral blood leukocytes. Cancer Res 52: 5 552s-5 553s

35. Murray PG, Young LS, Rowe M, Crocker J (1992) Immunohistochemical demonstration of the Epstein-Barr virus-encoded latent membrane protein in paraffin sections of Hodgkin's disease. J Pathol 166: 1-5

36. Okano M, Taguchi Y, Nakamine H, Pirruccello SJ, Davis JR, Beisel KW, Kleveland KL, Sanger WG, Fordyce RR, Purtilo DT (1990) Characterization of Epstein-Barr virusinduced lymphoproliferation derived from human peripheral blood mononuclear cells transferred to severe combined immunodeficient mice. Am J Pathol 137: 517-22

37. Pallesen G, Hamilton DS, Rowe M, Young LS (1991) Expression of Epstein-Barr virus latent gene products in tumour cells of Hodgkin's disease. Lancet 337: 320-322

38. Pisa P, Cannon MJ, Pisa EK, Cooper NR, Fox RI (1992) Epstein-Barr virus induced lymphoproliferative tumors in severe combined immunodeficient mice are oligoclonal. Blood 79: 173-179

39. Qu L, Rowe D (1992) Epstein-Barr virus latent gene expression in uncultured peripheral blood lymphocytes. J Virol 66: 3 715-3 724

40. Rochford R, Hobbs MV, Garnier JL, Cooper NR, Cannon MJ (1993) Plasmacytoid differentiation of Epstein-Barr virus-transformed B cells in vivo is associated with reduced expression of viral latent genes. Proc Natl Acad Sci USA 90: 352-356

41. Rochford R, Mosier DE (1994) Immunobiology of Epstein-Barr virus-associated lymphomas. Clin Immunol Immunopathol 71: 256-259 
42. Rochford R, Mosier DE (1995) Differential Epstein-Barr virus gene expression in B-cell subsets recovered from lymphomas in SCID mice after transplantation of human peripheral blood lymphocytes. J Virol 69: 150-155

43. Rooney C, Taylor N, Countryman J, Jenson H, Kolman J, Miller G (1988) Genome rearrangements activate the Epstein-Barr virus gene whose product disrupts latency. Proc Natl Acad Sci USA 85: 9 801-9 805

44. Rowe M, Young LS, Crocker J, Stokes H, Henderson S, Rickinson AB (1991) EpsteinBarr virus (EBV)-associated lymphoproliferative disease in the SCID mouse model: implications for the pathogenesis of EBV- positive lymphomas in man. J Exp Med 173: 147-158

45. Sample J, Liebowitz D, Kieff E (1989) Two related Epstein-Barr virus membrane proteins are encoded by separate genes. J Virol 63: 933-937

46. Straus SE, Cohen JI, Tosato G, Meier J (1993) NIH conference. Epstein-Barr virus infections: biology, pathogenesis, and management [Review]. Ann Int Med 118: 45-58

47. Thomas JA, Hotchin NA, Allday MJ, Amlot P, Rose M, Yacoub M, Crawford DH (1990) Immunohistology of Epstein-Barr virus-associated antigens in B cell disorders from immunocompromised individuals. Transplantation 49: 944-953

48. Thorley-Lawson D, Nadler LM, Bhan AK, Schooley RT (1985) BLAST-2 [EBVCS], an early cell surface marker of human B cell activation, is superinduced by Epstein Barr virus. J Immunol 134: 3007-3012

49. Tierney RJ, Steven N, Young LS, Rickinson AB (1994) Epstein-Barr virus latency in blood mononuclear cells: analysis of viral gene transcription during primary infection and in the carrier state. J Virol 68: 7 374-7 385

50. Uckun F (1990) Regulation of human B-cell ontogeny. Blood 76: 1908-1923

51. Wu TC, Mann RB, Charache P, Hayward SD, Staal S, Lambe BC, Ambinder RF (1990) Detection of EBV gene expression in Reed-Sternberg cells of Hodgkin's disease. Int J Cancer 46: 801-804

52. Young L, Alfieri C, Hennessy K, Evans H, O’Hara C, Anderson KC, Ritz J, Shapiro RS, Rickinson A, Kieff E, Cohen JI (1989) Expression of Epstein-Barr virus transformationassociated genes in tissues of patients with EBV lymphoproliferative disease. N Engl J Med 321: 1 080-1 085

Authors' address: Dr. R. Longnecker, Department of Microbiology-Immunology, Northwestern University Medical School, 303 East Chicago Avenue, Chicago, IL 60611, U.S.A. 